\title{
Risk of Major Bleeding in Patients with Venous Thromboembolism Treated with Rivaroxaban or with Heparin and Vitamin K Antagonists
}

Running head: Major Bleeding with Rivaroxaban or LMWH-VKA in VTE

Di Nisio $\mathrm{M}^{1,2}$, PhD; Ageno $W^{3}$, MD; Rutjes $\mathrm{AWS}^{4,5}$, PhD; Pap $\mathrm{AF}^{6}$, MD; Büller $\mathrm{HR}^{2}$, $\mathrm{PhD}$

${ }^{1}$ Department of Medical, Oral and Biotechnological Sciences, University "G. D'Annunzio" of Chieti-Pescara, Chieti, Italy; ${ }^{2}$ Department of Vascular Medicine, Academic Medical Center, Amsterdam, Netherlands;

${ }^{3}$ Department of Clinical and Experimental Medicine, University of Insubria, Varese; ${ }^{4}$ Institute of Social and Preventive Medicine, University of Bern, Bern, Switzerland; ${ }^{5}$ Centre for Systematic Reviews, Foundation, University "G. D'Annunzio" of Chieti-Pescara, Chieti, Italy; ${ }^{6}$ Bayer Pharma AG, Wuppertal, Germany

Address for correspondence: Marcello Di Nisio, Department of Medical, Oral and Biotechnological Sciences, University "G. D'Annunzio" of Chieti-Pescara, via dei Vestini 31, 66013 Chieti, Italy; e-mail: mdinisio@unich.it. Phone: +39 328 3290020; Fax: +39 (0)871 357361

\section{Total Word Count:}

Abstract Word Count: 250

Number of online figures: 4

Number of tables: 6

Number of references: 33 
Grants or other financial support: There was no funding source for the present study.

\begin{abstract}
The study aim was to identify predictive factors for major bleeding in patients receiving the novel oral factor Xa inhibitor rivaroxaban or enoxaparin-vitamin K antagonists (VKAs) for the treatment of acute symptomatic venous thromboembolism.
\end{abstract}

We analyzed data from patients included in the phase III EINSTEIN DVT and EINSTEIN PE studies. Factors associated with major bleeding events were assessed with best subset variable selection using Cox proportional hazards regression model. Three time windows were considered, i.e. the initial 3 weeks, after the third week onwards, and the entire duration of the anticoagulant treatment. Model discrimination was estimated using the C-statistic and validated internally by bootstrap techniques.

Major bleeding occurred in 40 (1.0\%) of 4130 patients receiving rivaroxaban and in 72 (1.7\%) of 4116 receiving enoxaparin/VKAs, with $44 \%$ of the major bleeding events occurring in the first 3 weeks of treatment. Significant risk factors for major bleeding were older age, black race, low hemoglobin concentrations, active cancer, and antiplatelet or nonsteroidal anti-inflammatory drug therapy. The discrimination of the model for major bleeding was high for the first 3 weeks (C-statistic 0.73), from the fourth week onwards (C-statistic 0.68), and the entire period of anticoagulant treatment (C-statistic 0.74).

This analysis identified risk factors for major bleeding in patients receiving the novel oral anticoagulant rivaroxaban or enoxaparin/VKAs for the treatment of acute venous thromboembolism. The prognostic model based on the combination of identified risk factors 
may be informative to estimate the risk of major bleeding both during the initial and later phases of anticoagulation.

Keywords: hemorrhage, anticoagulants, thrombosis, risk factors, randomized controlled trial 


\section{Introduction}

Patients receiving anticoagulant treatment for venous thromboembolism (VTE) are at increased risk of bleeding events. ${ }^{1,2}$ Accurate detection of risk factors for major bleeding may identify patients who could benefit from more intensive care (e.g. hospitalization) in the acute phase and may influence other clinical decisions, such as duration of anticoagulation therapy for long-term secondary prevention of VTE (extended vs. shorter-term treatment).

Combining risk factors for major bleeding during anticoagulation therapy to develop predictive scores has been tested in several studies but with heterogeneous results. ${ }^{3-11}$ Possible explanations of this inconsistency could include the use of different definitions for bleeding, indications for anticoagulation, number and type of predictive variables analyzed, trial designs and size, quality of anticoagulation, and quality of statistical model building. Different diseases, co-morbid conditions, or co-medications could cause variation in drugs metabolism or predispose to bleeding events. ${ }^{12,13}$ Currently, there are no validated decision tools for stratifying the risk of bleeding in patients with VTE who are treated with anticoagulants. The recent American College of Chest Physicians (ACCP) guidelines on antithrombotic therapies proposed a prediction rule that stratifies patients into low, moderate, or high risk of bleeding. ${ }^{14}$ However, the ACCP rule, as other scores, has not yet been validated in clinical practice. In an attempt to externally validate a number of predictive scores, Donzé and colleagues found limited discriminative power of all the risk scores tested. ${ }^{15}$ Although conclusions were limited by the small size of the study (515 patients with 35 major bleeding events), this work underlines the uncertainty around the available scores. Similar conclusions were reached in another recent report. ${ }^{16}$ All bleeding prediction rules proposed thus far have been developed from groups of patients receiving vitamin $\mathrm{K}$ 
antagonists (VKAs) only, and there are no data on the risk factors for bleeding in patients receiving the novel oral anticoagulants.

The aim of this study was to identify risk factors for major bleeding events in patients receiving rivaroxaban or standard anticoagulant therapy with enoxaparin/VKAs for acute VTE who were enrolled in the phase III EINSTEIN DVT and EINSTEIN PE trials. ${ }^{17,18}$ Because the risk of bleeding is higher during the first 3 weeks after the start of anticoagulation, ${ }^{1}$ and the relevance of risk factors may differ in the first 3 weeks compared with subsequent weeks of anticoagulant treatment, we separately evaluated the impact of the identified factors during the initial 3 weeks of treatment, after the third week onwards, and for the entire follow-up period of the studies. The results of this study may impact on treatment decisions with the ultimate goal of improving safety in patients treated for deep vein thrombosis (DVT) or pulmonary embolism (PE).

\section{Methods}

Study Population and Interventions in the EINSTEIN DVT and EINSTEIN PE trials The study design and the characteristics of the patients included in the phase III EINSTEIN DVT and EINSTEIN PE studies have been described previously. ${ }^{17-19}$ Briefly, EINSTEIN DVT and EINSTEIN PE were randomized, open-label studies that compared the efficacy and safety of rivaroxaban with standard therapy consisting of enoxaparin and a VKA in patients with acute, symptomatic and objectively confirmed proximal DVT or PE.

Patients were assigned to receive oral rivaroxaban $15 \mathrm{mg}$ twice daily for the first 3 weeks, followed by 20 mg once daily for an intended 3, 6, or 12 months of treatment. Patients who 
were assigned to standard therapy received subcutaneous enoxaparin $(1.0 \mathrm{mg} / \mathrm{kg}$ of body weight twice daily) and either warfarin or acenocoumarol, started within 48 hours after randomization. Enoxaparin was discontinued when the international normalized ratio (INR) was 2.0 or more for 2 consecutive days and the patient had received at least 5 days of enoxaparin treatment. The dose of VKA was adjusted to maintain an INR of 2.0 to 3.0. In both groups, therapeutic doses of low-molecular-weight heparin, fondaparinux, or unfractionated heparin for up to 48 hours before randomization or a single dose of a VKA were allowed. Treatment with thrombectomy, a vena cava filter, or a fibrinolytic agent for the acute VTE were exclusion criteria, as were any contraindications listed in the labeling of enoxaparin, warfarin, or acenocoumarol. Additional exclusion criteria were another indication for a VKA; creatinine clearance $<30 \mathrm{~mL} / \mathrm{min}$; clinically significant liver disease; bacterial endocarditis; active bleeding or a high risk of bleeding contraindicating anticoagulant treatment; systolic blood pressure >180 mm Hg or diastolic blood pressure $>110$ mm Hg; childbearing potential without proper contraceptive measures, pregnancy, or breast-feeding; concomitant use of strong cytochrome P450 3A4 inhibitors (e.g., human immunodeficiency virus protease inhibitors or systemic ketoconazole) or inducers (e.g., rifampicin, carbamazepine, or phenytoin); participation in another experimental pharmacotherapeutic program within 30 days before screening; and a life-expectancy of $<3$ months.

\section{Study Outcomes}

The outcome of the present analysis was major bleeding, which was defined as in the original studies as clinically overt bleeding associated with a decrease in the hemoglobin level of $\geq$ $2.0 \mathrm{~g} / \mathrm{dL}$, bleeding that led to the transfusion of $\geq 2$ units of red cells, or bleeding that was 
intracranial or retroperitoneal, or occurring in another critical site, or that contributed to death. ${ }^{17,18}$ Patients with more than one bleeding event were counted only once.

\section{Study Variables and Statistical Considerations}

The analysis of major bleeding included events that occurred after randomization up to 2 days after stopping study treatment. Patients without major bleeding within this time window were censored at the last documented study treatment (+ 2 days, not later than the last study visit). The appropriate prognostic models were selected with best subset variable selection using Cox proportional hazards regression model, i.e. we selected the best model for each number of possible predictors and chose the model at which the difference in score statistics between the best models of number of predictors $\mathrm{k}$ and $\mathrm{k}+1$ is no longer statistically significant at a level of 0.05 (interaction terms with p-value $>0.10$ were omitted too). ${ }^{20}$ The discrimination of the model was assessed by calculating Harrell's C statistic ${ }^{21}$. The C statistic corrected for the optimism, or over-fitting, in predictive models was calculated using bootstrapped datasets to repeatedly quantify the degree of over-fitting in the model building process. ${ }^{21,22}$ The discrimination of the prognostic model was calculated for the initial 3 weeks of treatment, after the third week onwards, and for the entire follow-up period. The choice of 3 weeks was motivated by the protocol-mandated reduction in the dose of rivaroxaban after the first 3 weeks of treatment.

The following variables were identified as potentially associated with the occurrence of major bleeding and included in the full models on which the best subset selection process was based: treatment group (rivaroxaban versus enoxaparin/VKAs), gender, active cancer, hemoglobin, race (Caucasian, Black, Asian, other which included France where the recording of race is legally not allowed), age (and interaction with treatment group), hypertension in 
medical history, hospitalization for the index event (and interaction with treatment group), intensive care admission for the index event, diabetes in the medical history, cardiovascular disease in the medical history, chronic heart failure in the medical history, calculated creatinine clearance (and interaction with treatment group), platelet count, weight, antiplatelet or NSAID medication, index event (PE or DVT only). The model thus checked for interactions by testing whether the risk factors had different (separate) associations with major bleeding for rivaroxaban and enoxaparin/VKAs. All the above variables were used as recorded at baseline except for active cancer where post-baseline diagnosis of cancer was also considered. Active cancer at baseline was defined as a diagnosis of cancer that occurred within 6 months before enrolment, any treatment for cancer within the previous 6 months, or recurrent or metastatic cancer; active cancer during the study was defined as a new diagnosis of cancer or recurrence of cancer after randomization. ${ }^{23}$ Data on continuous variable were initially included as a single continuous measurements. The linear relationship between each continuous variable and major bleeding was tested by fitting fractional polynomials ${ }^{24}$ which showed no evidence of nonlinear association between age, hemoglobin, or creatinine clearance and major bleeding. When indicated, continuous variables were transformed to obtain better parameter estimates and to ensure that the Cox model converged. For this reason, the following categories were used: age per 10 years; creatinine clearance (Cockcroft and Gault) as normal (i.e. creatinine clearance $\geq 80 \mathrm{ml} / \mathrm{min}$ ), mildly (creatinine clearance 50 $79 \mathrm{ml} / \mathrm{min}$ ), moderately (creatinine clearance 30-49 ml/min), or severely (creatinine clearance $<30 \mathrm{ml} / \mathrm{min}$ ) impaired. ${ }^{25}$ The renal function categories were included in the Cox proportional-hazards models as single covariates. For weight, the best fit was obtained with the linear term weight per $100 \mathrm{~kg}$ and the power function $1 /\left(\right.$ weight per 100) ${ }^{2}$. . Missing data for weight, height, calculated creatinine clearance, and hemoglobin were estimated with 
multiple imputation technique based on a Markov chain Monte Carlo approach. ${ }^{26}$ We decided a priori to exclude patients with clinically overt bleeding associated with a decrease in hemoglobin level of $\geq 2.0 \mathrm{~g} / \mathrm{dL}$ when the bleeding event occurred within 24 hours of study inclusion. This was done in the attempt not to overestimate the prognostic weight of hemoglobin concentrations, which in these cases could represent a sign of a hidden bleeding event becoming symptomatic on the day of inclusion rather than a real marker of the event. For this reason, one subject was excluded from all models because of a major bleeding (hemoglobin drop $>2 \mathrm{~g} / \mathrm{dL})$ and a low hemoglobin value $(9.7 \mathrm{~g} / \mathrm{dL})$ on the day of randomization.

Firth’s penalized maximum likelihood estimation was performed in the final Cox proportional hazards models to reduce bias in the parameter estimates. Based on the respective final best Cox proportional hazard model the linear predictor $\mathrm{Z}_{\mathrm{i}} \beta$ can be calculated for a subject $\mathrm{i}$ (in the study or a future subject with given covariate vector $\mathrm{Z}_{\mathrm{i}}$ ) to predict the cumulative major bleeding rate at time point t (i.e. at day 21, at day 180 ) as follows:

$\left.F(t)=1-S(t)=1-S_{0}(t)^{\exp (Z \beta)}\right) \cdot$ where $t=21,180,180+21=201$ days respectively.

Prediction of major bleeding in the current population by other bleeding scores ${ }^{3,5-6,9,11-12}$ was done by calculating the individual score per patient, categorizing the score into 3 levels (e.g.low, intermediate and high risk), fitting Cox proportional hazard models and calculating Harrell’s C statistic.

The statistical analyses were performed with SAS version 9.2 and by STATA 11.2.

\section{Results}


The EINSTEIN DVT and EINSTEIN PE studies randomized 3449 and 4832 patients, respectively (8281 in total), 99\% of whom contributed to the analyses. For the present analysis, only patients who took at least one dose of study medication were included (safety population, $\mathrm{N}=8245$ ). For the analysis of all major bleeding events, we considered 8245 patients up to 3 weeks, and up to the end of actual study treatment. All 8060 patients who were not censored/did not have an event within the first 3 weeks were included in the analysis of major bleeding after the first 3 weeks of treatment.

The characteristics of the study population are reported in Table 1. In the enoxaparin/VKAs group, the median duration of enoxaparin treatment was 7.4 days (interquartile range [IQR] 5.8 to 10.1$)$. Overall, the INR was in the therapeutic range (2.0 to 3.0) for a mean of $62 \%$ of the time, above 3.0 for $16 \%$ of the time, and below 2.0 for $22 \%$ of the time. The time in therapeutic range varied from $56 \%$ (month 1) to $66 \%$ (months 7 to 12). Patients in the enoxaparin/VKAs group received anticoagulant treatment for a median of 182 days (IQR 178 to 266 days). Rivaroxaban was administered for a median of 183 days (IQR 179 to 269 days) and adherence to rivaroxaban was $>80 \%$ in $93 \%$ of patients.

Overall, major bleeding occurred in 40 (1.0\%) of 4130 patients receiving rivaroxaban and in 72 (1.7\%) of 4116 patients receiving enoxaparin/VKAs. Of these bleeding events, 49 occurred in the first 3 weeks (44\%), $16(0.3 \%)$ in the rivaroxaban group and $33(0.7 \%)$ in the enoxaparin/VKAs group. Table 2 describes the major bleeding events that occurred during the study. 


\section{Events During the First 3 Weeks of Treatment}

Of 8246 patients, 8219 (99.7\%) contributed to the analyses for the first 3 weeks of treatment. Table 3 shows the results of the multivariable analysis of the variables associated with major bleeding during the first 3 weeks of anticoagulant therapy.

The use of rivaroxaban was associated with a lower risk and enoxaparin/VKAs with a higher risk of major bleeding (HR 0.43; 95\% CI 0.23 to 0.80). The presence of active cancer and black race were associated with a significantly higher risk of major bleeding (HR 3.47; 95\% CI 1.79 to 6.70 , and HR 3.26; 95\% CI 1.15 to 9.23, respectively).

There was an inverse relationship between the risk of bleeding and hemoglobin concentrations (HR per each mg/dL 0.72; 95\% CI 0.62 to 0.84 ) or renal function (HR 0.62; 95\% CI 0.42 to 0.91). Antiplatelet and/or NSAID use doubled the risk (HR 2.07; 95\% CI 1.12 to 3.82). The risk of major bleeding seemed higher at low weight (below $50 \mathrm{~kg}$ ) and it was numerically driven by the major bleeding in the enoxaparin/VKA treatment group ${ }^{19}$. (see online Graph 1).There was no statistical evidence that the association between major bleeding and age, creatinine clearance categories, or weight was different between treatment groups (p for interaction $>0.10$ ). The prognostic model including all variables (Table 3) had a high discrimination, as shown by a Harrel's C statistic of 0.79 ( 0.73 after correcting for selection and estimating optimism by the bootstrap procedure). In a hypothetical Caucasian patient with no active cancer, no antiplatelet or NSAIDs medication, hemoglobin of $10 \mathrm{mg} / \mathrm{dL}$, weight of $50 \mathrm{~kg}$, creatinine clearance of 30-49 $\mathrm{mL} / \mathrm{min}$, the predicted probability of a major bleeding event at 21 days was $2.7 \%$ when receiving enoxaparin/VKAs and $1.2 \%$ if she was on rivaroxaban (See Online Table). 


\section{Events After The first 3 Weeks of Treatment}

Table 4 shows the variables associated with major bleeding after the first 3 weeks of anticoagulant therapy. The use of rivaroxaban was associated with a lower risk and enoxaparin with a higher risk of major bleeding (HR 0.60; 95\% CI 0.37 to 1.00). The incidence of major bleeding was increased by $45 \%$ every 10 years of age (HR 1.45; 95\% CI 1.22 to 1.74). There was a significant inverse association between hemoglobin and risk of bleeding (HR per each mg/dL 0.66; 95 \% CI 0.53 to 0.81) and some suggestion that this relationship was different between males and females ( $\mathrm{p}$ for interaction $=0.0663$, see online Graph 2). Male and female patients had similar risk of major bleeding if hemoglobin was $12 \mathrm{mg} / \mathrm{dL}$ or higher (HR 1.40 at $12 \mathrm{mg} / \mathrm{dL}$; 95\% CI: 0.78 to 2.52). Compared to Caucasian, black race carried an almost four fold increased risk (HR 3.93; 95\% CI 1.42 to 10.86).

There was no statistical evidence that the association between major bleeding and age, creatinine clearance categories, or weight was different between treatment groups ( $\mathrm{p}$ for interaction $>0.05)$. The Harrel's C statistic of the prediction model (Table 4) was 0.74 (0.68 after correcting for selection and estimating optimism by the bootstrap procedure). In a hypothetical Caucasian female patient with no cardiovascular disease in medical history, hemoglobin of $10 \mathrm{mg} / \mathrm{dL}$, the predicted probability of a major bleeding event at 201 days was $1.6 \%$ if she was receiving enoxaparin/VKAs and $1.0 \%$ if on rivaroxaban (See web link).

\section{Events During the Entire Treatment Period}

Table 5 shows the variables associated with major bleeding during the entire treatment period. Active cancer, black race, and use of antiplatelet or NSAIDs were all independently associated with the occurrence of major bleeding events. A lower risk was observed with Rivaroxaban and a higher risk with enoxaparin/VKAs (HR 0.50; 95\% CI 0.34 to 0.74). There 
was an inverse relationship between hemoglobin concentrations and major bleeding (HR per each $\mathrm{mg} / \mathrm{dL}$ 0.65; 95\% CI 0.56 to 0.75 ), with some statistical evidence that the effect of hemoglobin was different between males and females ( $\mathrm{p}$ for interaction= 0.0041, see online Graph 3). The risk of major bleeding increased by 33\% every 10 years of age (HR 1.33; 95\% CI 1.17 to 1.52). There was a suggestion that the risk of major bleeding is higher at low weight (below $50 \mathrm{~kg}$ ) and numerically driven by the major bleeding in the enoxaparin/VKAs treatment group ${ }^{19}$ (see online Graph 4). The Harrel's C statistic of the prediction model (Table 5) was 0.77 (0.74 after correcting for selection and estimating optimism by the bootstrap procedure). In a hypothetical 60 year old female patient with no active cancer, no antiplatelet or NSAIDs medication, weight of $50 \mathrm{~kg}$, hemoglobin of $10 \mathrm{mg} / \mathrm{dL}$, no index PE, the predicted probability of a major bleeding event at 180 days (6 months) was 3.8\%, if she was on enoxaparin/VKAs and 1.9\% if on rivaroxaban (See web link).

\section{Discussion}

This study identified risk factors for major bleeding in patients receiving the novel oral direct factor Xa inhibitor rivaroxaban or enoxaparin/VKAs for the treatment of acute VTE. The combination of these risk factors into a prognostic model showed a high discrimination both during the initial and subsequent phases of the anticoagulation therapy.

The incidence of bleeding events differs between the initial phase of treatment and the subsequent phase, with the risk being higher in the first weeks of anticoagulation. ${ }^{1}$ With the risk of bleeding changing over time, the importance of risk factors may vary depending on the timing since the start of anticoagulant treatment. Therefore, the discriminative ability of the model was separately analyzed for the first 3 weeks of anticoagulant treatment, after the 
third week, and for the entire follow-up period. A number of variables differed between the three models, with some being associated with major bleeding both in the initial and subsequent phase of anticoagulation and others being apparently relevant only in the shortterm phase or in the long-term phase. As an example the concomitant use of antiplatelet or NSAIDs was associated with a higher risk of bleeding especially during the first weeks of anticoagulation. These results confirm previous observations ${ }^{27}$ and suggest that in patients with a clinical indication for long-term NSAID or additional antiplatelet treatment, a dose reduction or interruption of these agents should be considered whenever possible. Another example was body weight, which was significantly associated with a higher risk of major bleeding only during the first 3 weeks. Black race emerged as a significant predictor of events in all treatment phases considered. The increased risk in black versus other races was largely driven by a higher incidence of major gastrointestinal bleeding in the enoxaparin/VKAs group (3.6\% in Black race patients vs. 0.4\% in Caucasian patients). Previous research did not report race as predictor, so that we cannot exclude that black race is a confounding indicator, highly associated with an unknown predictor that we did not consider in our models.

The development of a model to estimate the risk of major bleeding in patients receiving anticoagulant therapy for VTE has been the objective of intensive investigation. ${ }^{3-11}$ One of the most studied predictive scores is the Outpatient Bleeding Risk Index (OBRI), which was retrospectively derived from a group of 627 hospitalized patients, of whom only 103 (17\%) received treatment for VTE. ${ }^{5,6}$ The OBRI score was externally validated by Wells and colleagues, who suggested a good discrimination between low- and moderate-risk patients, although results for the high-risk group were inconclusive because this subgroup was underrepresented in the study. ${ }^{28}$ Most risk scoring systems are limited by a retrospective study design ${ }^{7,9,12,29}$ and/or lack of external or prospective validation. ${ }^{9,29}$ In a recent cohort of 
515 internal medicine patients receiving VKAs, Donzé and colleagues prospectively validated seven predictive scores. ${ }^{15}$ Their findings suggested poor discrimination of all scores, as indicated by calculated C statistic values between 0.54 and 0.61 , with no significant differences between the scores. The authors found that the performance of the scores was not statistically better than the physicians' subjective risk assessment, and only the ATRIA score performed slightly better than expected by chance alone. Similar results were obtained in two other studies comparing the clinical performance of several bleeding risk tools. ${ }^{16,30,31}$ In the current study, the $\mathrm{C}$ statistic of the model varied from 0.74 for the entire period of anticoagulation and the first 3 weeks to 0.68 from the fourth week onwards. These C statistic values indicate good performance and seem to compare favorably with other models. Table 6 depicts results of the current prediction model and of the external validation of previously published scores in terms of the Harrel's C statistics with 95\% confidence intervals. The EINSTEIN DVT and EINSTEIN PE study populations were used in all these analyses. However, the indirectness of the comparison with differences in study population and type of variables included in the model limits conclusions and external validation of our prediction score is desired.

\section{Limitations}

The major limitation of this study is related to the applicability of the model, given that in a phase III trial patients with contraindications to anticoagulant therapy are excluded, thus making the present findings not generalizable to the whole population of patients with VTE. Some factors significantly associated with bleeding in previous scores, such as hepatic failure, active gastroduodenal ulcer, gastrointestinal bleeding, severe renal insufficiency, and alcohol abuse were exclusion criteria for the EINSTEIN studies. As a consequence, we were 
not able to assess the importance of these factors in our risk score. In the external validation of other bleeding risk scores such as the OBRI or HAS-BLED, we had to appoint a "0" value representing the absence of such risk factor, which may have affected the calculated statistics, so that the descriptive comparison between any of the models displayed in Table 6 should be considered exploratory. The overall incidence of major bleeding in this study was $1.3 \%$, which compares well with the rates reported in other randomized clinical trials, ${ }^{1,32}$ but appears to be slightly lower than that reported in observational cohort studies. $3,4,7,13,15$ Although our analyses included a large number of patients, the total number of major bleeding events is still rather small and bootstrapping internal validation might only mitigate the effect of low statistical power. External validation of these findings in an independent and sufficiently large dataset is warranted.

We did not have information about genetic risk factors for major bleeding such as CYP 2C9 mutations, although the value of genetic testing for the prediction of bleeding risk is still controversial and not widely used in clinical practice. ${ }^{1,33}$

\section{Strengths}

This is one of the first studies that attempted to identify bleeding risk factors for one of the novel oral anticoagulants, namely the factor Xa inhibitor rivaroxaban. When possible, the analysis did not merely consider the presence or absence of risk factors, but rather took into account their severity (e.g. platelet count, renal function, age), which may influence the risk of bleeding. The current analysis identified a small number of risk factors readily available from the clinical medical history or routinely tested in patients with VTE which could be used to calculate the risk of major bleeding in an individual patient $i$ at a time $t$ by an userfriendly online tool (See Online Table). Finally, major strengths of the study are the relatively 
large sample size, the completeness of the dataset, and the adjudication of all bleeding end points, including major bleeding by an external blinded independent committee, thus reducing the risk of assessment bias.

We identified risk factors for major bleeding in patients receiving rivaroxaban for the treatment of acute VTE. The bleeding prognostic model based on the combination of identified risk factors showed high discrimination. Future studies are warranted to validate this model and to confirm an approach stratified by the time elapsed after the start of anticoagulation.

\section{Acknowledgments}

We are very grateful to Martin Homering and Martin Gebel from Bayer Pharma AG, Wuppertal, Germany for helping with the statistical analyses.

\section{Contributions}

Study concept and design: Di Nisio, Ageno, Rutjes, Büller; Analysis and interpretation of data: Di Nisio, Ageno, Rutjes, Pap, Büller; Drafting of the manuscript: Di Nisio, Ageno, Rutjes, Büller; Critical revision of the manuscript for important intellectual content: Di Nisio, Ageno, Rutjes, Pap, Büller; Final approval of the article: Di Nisio, Ageno, Rutjes, Pap, Büller. 


\section{Conflict of Interest Disclosures}

Drs Di Nisio, Ageno and Büller have received financial compensation for consultancy from Bayer HealthCare, ÁF Pap is an employee of the Bayer Pharma AG.

There was no funding source for the present study. The EINSTEIN DVT and EINSTEIN PE were supported by Bayer HealthCare and Janssen Pharmaceuticals. 


\section{References}

34. Ageno W, Gallus AS, Wittkowsky A, et al. Oral anticoagulant therapy : antithrombotic therapy and prevention of thrombosis, 9th ed: American College of Chest Physicians Evidence-Based Clinical Practice Guidelines. Chest. 2012;141;e44S-e88S.

35. Linkins LA, Choi PT, Douketis JD. Clinical impact of bleeding in patients taking oral anticoagulant therapy for venous thromboembolism: a meta-analysis. Ann Intern Med. 2003;139:893-900.

36. Ruíz-Giménez N, Suárez C, González R, et al. Predictive variables for major bleeding events in patients presenting with documented acute venous thromboembolism. Findings from the RIETE Registry. Thromb Haemost. 2008;100:26-31.

37. Nieto JA, Solano R, Ruiz-Ribó MD, et al. Fatal bleeding in patients receiving anticoagulant therapy for venous thromboembolism: findings from the RIETE registry. J Thromb Haemost. 2010;8:1216-1222.

38. Landefeld CS, Goldman L. Major bleeding in outpatients treated with warfarin: incidence and prediction by factors known at the start of outpatient therapy. Am $J$ Med. 1989;87:144-152.

39. Landefeld CS, Cook EF, Flatley M, et al. Identification and preliminary validation of predictors of major bleeding in hospitalized patients starting anticoagulant therapy. Am J Med. 1987;82:703-713.

40. Beyth BJ, Quinn LM, Landefeld CS. Prospective evaluation of an index for predicting the risk of major bleeding in outpatients treated with warfarin. Am J Med. 1998;105:91-99. 
41. Nieuwenhuis HK, Albada J, Banga JD, et al. Identification of risk factors for bleeding during treatment of acute venous thromboembolism with heparin or low molecular weight heparin. Blood. 1991;78:2337-2343.

42. Kuijer PMM, Hutten BA, Prins MH, et al. Prediction of the risk of bleeding during anticoagulant treatment for venous thromboembolism. Arch Intern Med. 1999;159:457-460.

43. Kearon C, Ginsberg JS, Kovacs MJ, et al, for the Extended Low-Intensity Anticoagulation for Thrombo-Embolism Investigators. Comparison of low-intensity warfarin therapy with conventional-intensity warfarin therapy for long-term prevention of recurrent venous thromboembolism. N Engl J Med. 2003;349:631-639.

44. Pisters R, Lane DA, Nieuwlaat R, de Vos CB, Crijns HJ, Lip GY.A novel userfriendly score (HAS-BLED) to assess 1-year risk of major bleeding in patients with atrial fibrillation: the Euro Heart Survey. Chest. 2010;138:1093-100.

45. Gage BF, Yan Y, Milligan PE, et al. Clinical classification schemes for predicting hemorrhage: Results from the National Registry of Atrial Fibrillation (NRAF). Am Heart J. 2006;151:713-719.

46. Poli D, Antonucci E, Testa S, et al; for the Italian Federation of Anticoagulation Clinics (FCSA). Bleeding risk in very old patients on vitamin K antagonist treatment: results of a prospective collaborative study on elderly patients followed by Italian Centres for Anticoagulation. Circulation. 2011;124:824-829.

47. Kearon C, Akl EA, Comerota AJ, et al. Antithrombotic therapy for VTE disease. Antithrombotic therapy and prevention of thrombosis, 9th ed: American College of Chest Physicians Evidence-Based Clinical Practice Guidelines. Chest. 2012;141(2)(Suppl);e419S-494S. 
48. Donzé J, Rodondi N, Waeber G, et al. Scores to predict major bleeding risk during oral anticoagulation therapy: a prospective validation study. Am J Med. 2012;125:1095-1102.

49. Riva N, Bellesini M, Di Minno MND, et al. Poor predictive value of contemporary bleeding risk scores during long-term treatment of venous thromboembolism A multicentre retrospective cohort study. Thromb Haemost 2014; 112: 511-521.

50. The EINSTEIN Investigators. Oral rivaroxaban for symptomatic venous thromboembolism. N Engl J Med. 2010;363:2499-2510.

51. The EINSTEIN-PE Investigators. Oral rivaroxaban for the treatment of symptomatic pulmonary embolism. $N$ Engl J Med. 2012;366:1287-1297.

52. Prins MH, Lensing AWA, Bauersachs R, et al, for the EINSTEIN Investigators. Oral rivaroxaban versus standard therapy for the treatment of symptomatic venous thromboembolism: a pooled analysis of the EINSTEIN-DVT and PE randomized studies. Thromb J. 2013;11:21. Doi: 10.1186/1477-9560-11-21.

53. Hosmer (Jr) DW, Lemeshow S, Sturdivant RX. Applied Logistic Regression. 3rd Edition. Wiley Series in Probability \& Statistics; 2003.

54. Harrell FE Jr, Lee KL, Mark DB. Multivariable prognostic models: issues in developing models, evaluating assumptions and adequacy, and measuring and reducing errors. Stat Med. 1996;15:361-87.

55. Miao Y, Stijacic Cenzer I, Boscardin J. Estimating Harrell’s Optimism on Predictive Indices Using Bootstrap Samples. Paper 504-2013. SAS Global Forum 2013.

56. Prins MH, Lensing AWA, Brighton TA, et al. Oral rivaroxaban versus enoxaparin with vitamin $\mathrm{K}$ antagonist for the treatment of symptomatic venous thromboembolism in patients with cancer (EINSTEIN-DVT and EINSTEIN-PE): a pooled subgroup 
analysis of two randomised controlled trials. The Lancet Haematology 2014; 1: e37e46.

57. Royston P, W. Sauerbrei W. Multivariable Model-building: A Pragmatic Approach to Regression Analysis Based on Fractional Polynomials for Modelling Continuous Variables; Chichester, UK: Wiley; 2008.

58. Bauerschs RM, Lensing AWA, Prins MH, et al. Rivaroxaban versus enoxaparin/vitamin K antagonist therapy in patients with venous thromboembolism and renal impairment. Thrombosis Journal 2014; 12:25. Doi:10.1186/1477-9560-1225

59. Schafer JL. Analysis of Incomplete Multivariate Data. London, United Kingdom: Chapman \& Hall; 1997.

60. Davidson BL, Verheijen S, Lensing AWA, et al. Bleeding risk of patients with acute venous thromboembolism taking nonsteroidal anti-inflammatory drugs or aspirin. JAMA Intern Med. 2014:174:947-953.

61. Wells PS, Forgie MA, Simms M, et al. The outpatient bleeding risk index validation of a tool for predicting bleeding rates in patients treated for deep venous thrombosis and pulmonary embolism. Arch Intern Med. 2003;163:917-920.

62. Fang MC, Go AS, Chang Y, et al. A new risk scheme to predict warfarin-associated hemorrhage. The ATRIA (Anticoagulation and Risk Factors in Atrial Fibrillation) Study. J Am Coll Cardiol. 2011;58:395-401.

63. Burgess S, Crown N, Louzada ML, et al. Clinical performance of bleeding risk scores for predicting major and clinically relevant non-major bleeding events in patients receiving warfarin. J Thromb Haemost. 2013;11:1647-1654. 
64. Scherz N, Méan M, Limacher A, et al. Prospective, multicenter validation of prediction scores for major bleeding in elderly patients with venous thromboembolism. J Thromb Haemost. 2013;11:435-443.

65. Schulman S, Beyth RJ, Kearon C, et al. Hemorrhagic complications of anticoagulant and thrombolytic treatment: American College of Chest Physicians Evidence-Based Clinical Practice Guidelines (8th Edition). Chest. 2008;133;257-298.

66. Olesen JB, Lip GYH, Hansen PR, et al. Bleeding risk in "real world" patients with atrial fibrillation: comparison of two established bleeding prediction schemes in a nationwide cohort. J Thromb Haemost. 2011;9:1460-1467. 


\section{Tables}

\section{Table 1. Demographic Data and Baseline Characteristics}

\begin{tabular}{lccc}
\hline & Rivaroxaban & Enoxaparin/VKA & Total \\
& $(\mathrm{N}=4130)$ & $(\mathrm{N}=4116)$ & $(\mathrm{N}=8246)$ \\
\hline Age at enrollment, years, mean (SD) & $57.0(17.0)$ & $57.0(16.8)$ & $57.0(16.9)$ \\
$<65$ years, $\mathrm{n}(\%)$ & $2592(62.8)$ & $2579(62.7)$ & $5171(62.7)$ \\
65 to 75 years, $\mathrm{n}(\%)$ & $883(21.4)$ & $913(22.2)$ & $1796(21.8)$ \\
$>75$ years, $\mathrm{n}(\%)$ & $655(15.9)$ & $624(15.2)$ & $1279(15.5)$
\end{tabular}

Sex, n (\%)

Males

Females

Race, n (\%)
$2898(70.2)$

$104(2.5)$
$2294(55.5)$

1836 (44.5)

Black
5794 (70.3)

215 (2.6) 


\begin{tabular}{cccc}
\hline & Rivaroxaban & Enoxaparin/VKA & Total \\
& $(\mathrm{N}=4130)$ & $(\mathrm{N}=4116)$ & $(\mathrm{N}=8246)$ \\
\hline Asian & $388(9.4)$ & $371(9.0)$ & $759(9.2)$ \\
Other & $740(17.9)$ & $738(17.9)$ & $1478(17.9)$
\end{tabular}

Body mass index $\left(\mathrm{kg} / \mathrm{m}^{2}\right)$

$\begin{array}{lccc}\text { Mean (SD) } & 28.1(5.7) & 28.1(5.6) & 28.1(5.7) \\ <30 \mathrm{~kg} / \mathrm{m}^{2}, \mathrm{n}(\%) & 2860(69.2) & 2857(69.4) & 5717(69.3) \\ \geq 30 \mathrm{~kg} / \mathrm{m}^{2}, \mathrm{n}(\%) & 1248(30.2) & 1235(30.0) & 2483(30.1) \\ \text { Creatinine clearance* } & & & \\ \text { Mean (SD) } & 101.8(44.3) & 101.8(41.6) & 5543(67.2) \\ \geq 80 \mathrm{~mL} / \mathrm{min} & 2757(66.8) & 2786(67.7) & 2026(24.6) \\ 50 \mathrm{to}<80 \mathrm{~mL} / \mathrm{min} & 1027(24.9) & 999(24.3) & 650(7.9)\end{array}$




\begin{tabular}{|c|c|c|c|}
\hline & $\begin{array}{l}\text { Rivaroxaban } \\
(\mathrm{N}=4130)\end{array}$ & $\begin{array}{c}\text { Enoxaparin/VKA } \\
(\mathrm{N}=4116)\end{array}$ & $\begin{array}{c}\text { Total } \\
(\mathrm{N}=8246)\end{array}$ \\
\hline \multicolumn{4}{|l|}{ Index event, n (\%) } \\
\hline Only DVT & $1688(40.9)$ & $1687(41.0)$ & 3375 (40.9) \\
\hline PE with DVT & $615(14.9)$ & $595(14.5)$ & $1210(14.7)$ \\
\hline PE without DVT & $1787(43.3)$ & $1798(43.7)$ & $3585(43.5)$ \\
\hline No confirmed index event & $40(1.0)$ & $36(0.9)$ & $76(0.9)$ \\
\hline \multicolumn{4}{|l|}{ Cause of DVT/PE, n (\%) } \\
\hline Spontaneous DVT/PE & $2605(63.1)$ & $2628(63.8)$ & $5233(63.5)$ \\
\hline Secondary DVT/PE & 1525 (36.9) & $1488(36.2)$ & $3013(36.5)$ \\
\hline Recent surgery or trauma & $752(18.2)$ & $726(17.6)$ & 1478 (17.9) \\
\hline Immobilization & $648(15.7)$ & $636(15.5)$ & $1284(15.6)$ \\
\hline Use of estrogen-containing drugs & $345(8.4)$ & $337(8.2)$ & $682(8.3)$ \\
\hline
\end{tabular}




\begin{tabular}{lccc}
\hline & Rivaroxaban & Enoxaparin/VKA & Total \\
& $(\mathrm{N}=4130)$ & $(\mathrm{N}=4116)$ & $(\mathrm{N}=8246)$ \\
\hline Puerperium & $12(0.3)$ & $15(0.4)$ & $27(0.3)$ \\
Active cancer & $232(5.6)$ & $196(4.8)$ & $428(5.2)$ \\
Cancer status, n (\%) & & & \\
No active cancer & $3814(92.3)$ & $3838(93.2)$ & $7652(92.8)$ \\
Active cancer at baseline & $232(5.6)$ & $196(4.8)$ & $428(5.2)$ \\
Cancer reported as adverse event during the study & $84(2.0)$ & $82(2.0)$ & $166(2.0)$
\end{tabular}

Diabetes, n (\%)

$\begin{array}{llll}\text { No } & 3719(90.0) & 3692(89.7) & 7411(89.9) \\ \text { Yes } & 411(10.0) & 424(10.3) & 835(10.1)\end{array}$

Chronic heart failure, n (\%)

No

4057 (98.2)

$4064(98.7)$

$8121(98.5)$ 
Published in final edited form as: Thromb Haemost. 2016 Jan;115(2):424-32. doi: 10.1160/TH15-06-0474

\begin{tabular}{cccc}
\hline & Rivaroxaban & Enoxaparin/VKA & $(\mathrm{N}=4116)$ \\
& $(\mathrm{N}=4130)$ & & \\
\hline Yes & $73(1.8)$ & $52(1.3)$
\end{tabular}

Cardiovascular disease, $\mathrm{n}(\%)$

No

Yes

Previous episode(s) of DVT/PE, n (\%)

Previous episode(s) of DVT/PE

No previous episode(s) of DVT/PE

Pre-randomization LMWH treatment taken, n (\%)

No pre-randomization treatment

Pre-randomization treatment given
3787 (91.7)

$343(8.3)$

$788(19.1)$

$3342(80.9)$

$645(15.6)$

$3485(84.4)$
3807 (92.5)

$7594(92.1)$

$309(7.5)$

$652(7.9)$

1601 (19.4)

813 (19.8)

6645 (80.6)

3303 (80.2)

$688(16.7) \quad 1333(16.2)$

$3428(83.3) \quad 6913(83.8)$ 


\begin{tabular}{|c|c|c|c|}
\hline & $\begin{array}{l}\text { Rivaroxaban } \\
(\mathrm{N}=4130)\end{array}$ & $\begin{array}{c}\text { Enoxaparin/VKA } \\
\qquad(\mathrm{N}=4116)\end{array}$ & $\begin{array}{c}\text { Total } \\
(\mathrm{N}=8246)\end{array}$ \\
\hline \multicolumn{4}{|l|}{ Duration of (LMWH/heparin/fondaparinux) } \\
\hline pre-medication (days) & & & \\
\hline Median & 1.00 & 1.00 & 1.00 \\
\hline Min & 0.5 & 0.5 & 0.5 \\
\hline Max & 37.0 & 6.8 & 37.0 \\
\hline \multicolumn{4}{|l|}{ Hemoglobin at baseline (g/dL), $\mathrm{n}(\%)$} \\
\hline Mean (SD) & $13.5(1.8)$ & $13.5(1.7)$ & $13.5(1.7)$ \\
\hline$\leq 10 \mathrm{~g} / \mathrm{dL}$ & $148(3.6)$ & $155(3.8)$ & $303(3.7)$ \\
\hline Females: 10 to $<12 \mathrm{~g} / \mathrm{dL}$, Males: 10 to $<13 \mathrm{~g} / \mathrm{dL}$ & $768(18.6)$ & $725(17.6)$ & $1493(18.1)$ \\
\hline Females: $\geq 12 \mathrm{~g} / \mathrm{dL}$, Males: $\geq 13 \mathrm{~g} / \mathrm{dL}$ & 3205 (77.6) & 3219 (78.2) & $6424(77.9)$ \\
\hline
\end{tabular}




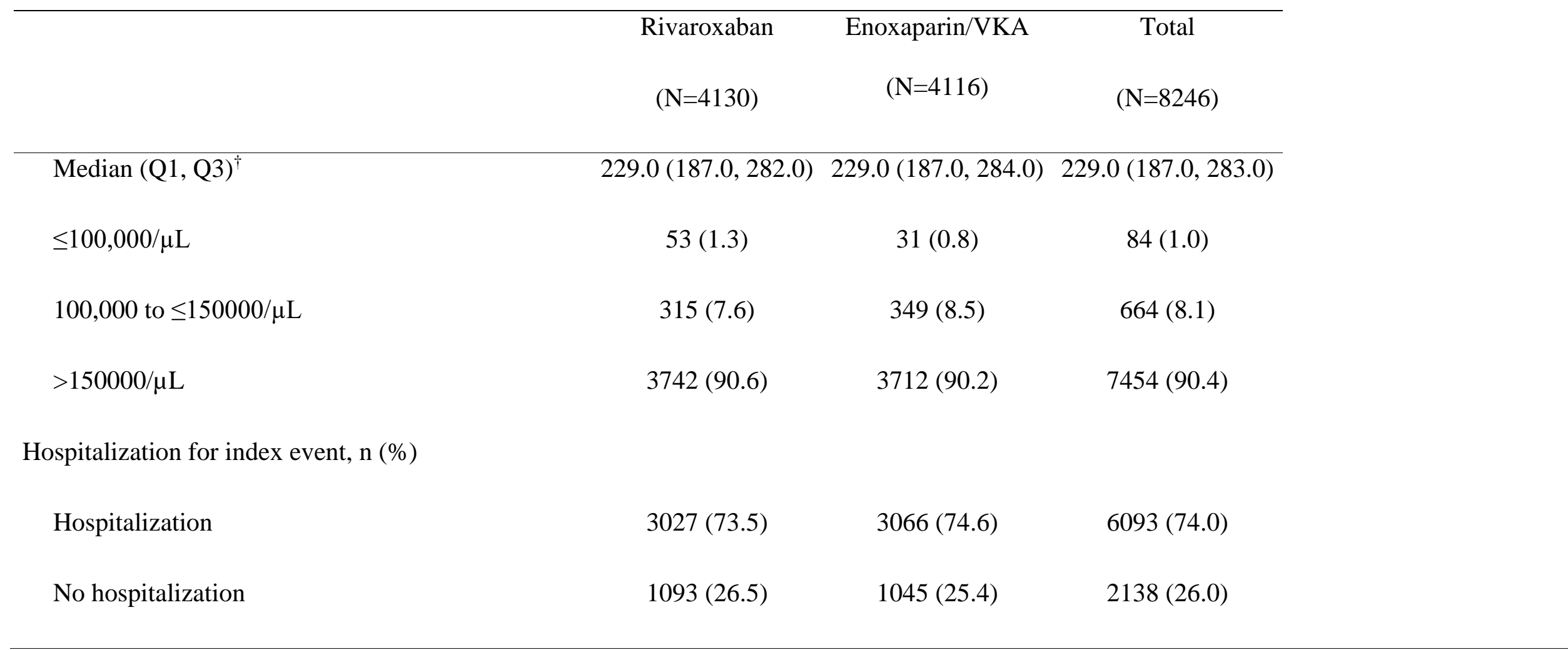

* Creatinine clearance levels were calculated using the equation of Cockroft and Gault, using the last value up to the day of randomization.

† Q1 = 25th percentile, Q3 = 75th percentile. AE indicates adverse event; LMWH, low-molecular-weight heparin; DVT, deep vein thrombosis; PE, pulmonary embolism; SD, standard deviation; and VKA, vitamin K antagonist. 


\section{Table 2. Incidence of Major Bleeding in the Safety Population}

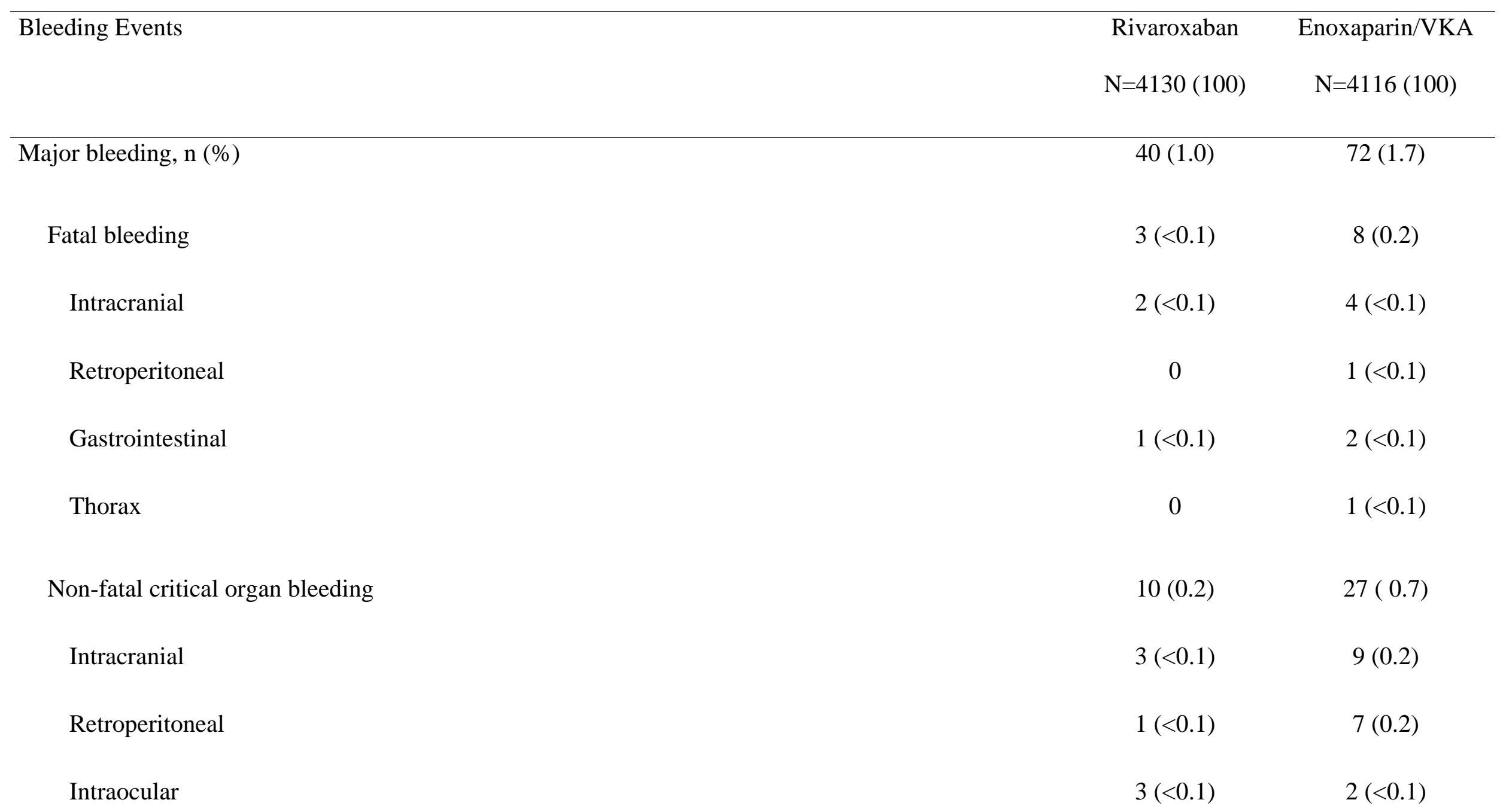


Pericardial

Intra-articular

Adrenal gland

Pulmonary

Abdominal

Vitreous body

Non-fatal non-critical organ bleeding (fall in $\mathrm{Hb} \geq 2 \mathrm{~g} / \mathrm{dl}$ and/or transfusions $\geq 2$ units)

Surgical site

Skin (other than injection site)

Urogenital

Gastrointestinal
0

$2(<0.1)$

0

$4(<0.1)$

$1(<0.1)$

0

$1(<0.1)$

$1(<0.1)$

0

$1(<0.1)$

$27(0.7)$

$36(0.9)$

$3(<0.1)$

$1(<0.1)$

$4(<0.1)$

$2(<0.1)$

$12(0.3)$

$20(0.5)$ 


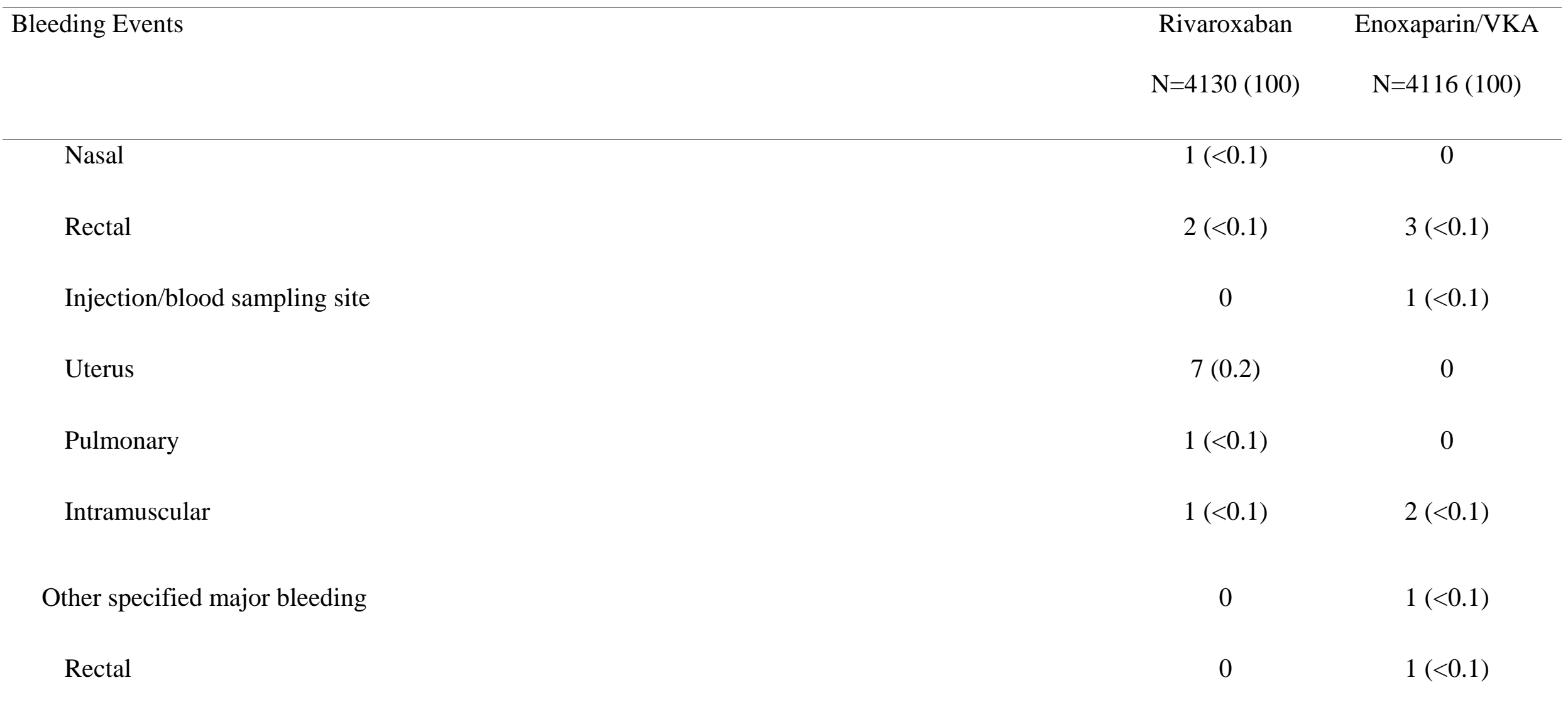

Hb indicates hemoglobin; and VKA, vitamin K antagonist 
Table 3. Risk Factors for Major Bleeding Events in the First 3 Weeks of Treatment

Covariate

Hazard Ratio (95\% CI)

Anticoagulant Treatment

Rivaroxaban vs. Enoxaparin/VKA

$0.43(0.23$ to 0.80$)$

Active cancer

3.47 (1.79 to 6.70$)$

Hemoglobin, mg/dl

$0.72(0.62$ to 0.84$)$

Race

Black vs. Caucasian

3.26 (1.15 to 9.23)

Asian vs. Caucasian

$1.01(0.38$ to 2.71$)$

Other vs. Caucasian

$1.78(0.87$ to 3.65$)$

Antiplatelet and/or NSAID concomitant medication at baseline*

$2.07(1.12$ to 3.82$)$

Weight, per $100 \mathrm{~kg}$

18.56 (1.81 to 190.6$)$

Weight in $1 /(\text { Weight per100 })^{2}$

1.69 (1.01 to 2.80$)$ 
* vs. no such concomitant medication; †Renal function was categorized as normal (i.e. $\mathrm{CrCl} \geq 80 \mathrm{ml} / \mathrm{min}$; score: 3 ), or with impairment that was mild ( $\mathrm{CrCl}$ 50-79 ml/min; score: 2), moderate ( $\mathrm{CrCl} 30-49 \mathrm{ml} / \mathrm{min}$; score: 1$)$, or severe $(\mathrm{CrCl}<30 \mathrm{ml} / \mathrm{min}$; score: 0$)$. CI indicates confidence interval; NSAIDs, non-steroidal anti-inflammatory drugs; and VKA, vitamin K antagonist. 
Table 4. Risk Factors for Major Bleeding Events After the First 3 Weeks of Treatment

Covariate Hazard Ratio (95\% CI)

Anticoagulant Treatment

Rivaroxaban vs. Enoxaparin/VKA

$0.60(0.37$ to 1.00$)$

Age, per 10 years

1.45 (1.22 to 1.74$)$

Hemoglobin, mg/dl

$0.66(0.53$ to 0.81$)$

Male sex if hemoglobin $12 \mathrm{mg} / \mathrm{dl}$

$1.40(0.78$ to 2.52$)$

Hemoglobin among males, mg/dl

$1.31(0.98$ to 1.74$)$

Race

Black vs. Caucasian

Asian vs. Caucasian

1.11 (0.44 to 2.82$)$

Other vs. Caucasian

1.74 (0.98 to 3.07$)$

Cardiovascular disease in the medical history

1.74 (0.91 to 3.33$)$ 
CI indicates confidence interval and VKA, vitamin K antagonist

Table 5. Risk Factors for Major Bleeding Events in the Entire Study Treatment Period

Covariate

Hazard Ratio (95\% CI)

Anticoagulant Treatment

Rivaroxaban vs. Enoxaparin/VKA

$0.50(0.34$ to 0.74$)$

Active cancer

2.49 (1.54 to 4.03$)$

Hemoglobin, mg/dl

0.65 (0.56 to 0.75$)$

Male sex if hemoglobin $12 \mathrm{mg} / \mathrm{dl}$

$1.05(0.65$ to 1.71$)$

Hemoglobin among males, mg/dL

1.37 (1.11 to 1.70$)$

Race

Black vs. Caucasian

2.84 (1.23 to 6.55$)$

Asian vs. Caucasian

1.03 (0.51 to 2.07 )

Other vs. Caucasian

1.54 (0.97 to 2.44$)$ 
Antiplatelet and/or NSAIDs concomitant medication at baseline*

Age, per 10 years

Weight, per $100 \mathrm{~kg}$

Weight in $1 /\left(\right.$ Weight per100) ${ }^{2}$

Index PE (yes vs. no)
1.75 (1.16 to 2.64$)$

1.33 (1.17 to 1.52$)$

3.24 (0.45 to 23.26$)$

1.48 (0.99 to 2.21$)$

$1.48(0.97$ to 2.27$)$

* vs. no such concomitant medication; CI indicates confidence interval; NSAIDs, non-steroidal anti-inflammatory drugs; and VKA, vitamin K antagonist. 
Table 6. Prediction of major bleeding in the current population by other bleeding scores

\begin{tabular}{|l|l|l|l|}
\hline Bleeding index/score & First 3 Weeks of Treatment & Fourth week onwards & Entire Study Treatment Period \\
\hline Current Prediction model & $0.73(0.65$ to 0.81$)$ & $0.68(0.60$ to 0.76$)$ & $0.74(0.68$ to 0.80$)$ \\
\hline Outpatient bleeding risk index score 5 ,6 & $0.54(0.49$ to 0.58$)$ & $0.53(0.49$ to 0.56$)$ & $0.53(0.50$ to 0.56$)$ \\
\hline Kuijer score $^{9}$ & $0.64(0.57$ to 0.72$)$ & $0.64(0.60$ to 0.68$)$ & $0.64(0.60$ to 0.68$)$ \\
\hline HEMORR2HAGES score ${ }^{12}$ & $0.64(0.57$ to 0.72$)$ & $0.66(0.59$ to 0.72$)$ & $0.65(0.60$ to 0.70$)$ \\
\hline HAS-BLED score ${ }^{11}$ & $0.58(0.51$ to 0.64$)$ & $0.57(0.52$ to 0.62$)$ & $0.59(0.55$ to 0.64$)$ \\
\hline RIETE score & & $0.63(0.60$ to 0.66$)$ & 0.60 (0.57 to 0.63$)$ \\
\hline
\end{tabular}


The table depicts results of the current prediction model and of external validations of previously published scores categorized into 3 levels (e.g. low, intermediate and high risk) in terms of the Harrel's C statistics with 95\% confidence intervals.The EINSTEIN DVT and EINSTEIN PE study populations were used in all analyses. 
Published in final edited form as: Thromb Haemost. 2016 Jan;115(2):424-32. doi: 10.1160/TH15-06-0474 